\section{Hacia una formación integrada en la currícula universitaria: el valor de la práctica en la Universidad Nacional de La Pampa}

María Graciela Di Franco

chdifarnco@gmail.com

Valeria Alfageme Balza

vale.alfageme@gmail.com

\section{Norma Di Franco}

ndifranco@hotmail.com

Docentes investigadoras de la Facultad

de Ciencias Humanas. Universidad

Nacional de La Pampa, Argentina.
Integración de la docencia y la extensión /

Desafíos de gestión

RECEPCIÓN: 24/06/16

ACEPTACIÓN FINAL: 15/10/16

\section{Resumen}

Las autoras de este trabajo coordinamos actividades de extensión en el contexto de la Universidad Nacional de La Pampa (UNLPam). En este artículo nos proponemos, en primer lugar, poner en contexto prácticas de extensión en escuelas y comunidades alejadas del centro de la ciudad en relación a educación sexual integral y acompañamiento a adolescentes embarazadas con trayectorias escolares en riesgo. En un segundo momento, reflexionar acerca de estas prácticas de extensión desde el marco normativo de la UNLPam y de la importancia de su curricularización. Y en el tercer y último apartado, analizar acerca de la necesidad de integrar las distintas prácticas que prescriben las currículas universitarias: prácticas de extensión; prácticas profesionales y prácticas sociocomunitarias para favorecer una formación integrada, potente y políticamente comprometida en la comprensión e intervención social.
Palabras-clave

- Práctica

- Integración

- Curriculum

- Praxis

\section{Resumo}

Os autores deste trabalho coordenamos atividades de extensão no âmbito da Universidad Nacional de La Pampa (UNLPam). Neste artigo nos propomos, em primeiro lugar, contextualizar práticas de extensão em escolas e comunidades afastadas do centro da cidade quanto à educação sexual integral e apoio para adolescentes grávidas com trajetórias escolares em risco. Em um segundo momento, refletir sobre estas práticas extensão no âmbito normativo da UNLPam e sobre a importância da sua curricularização. E no terceira e última etapa, analisar sobre a necessidade de integrar as diversas práticas que prescrevem os currículos universitários: práticas de extensão; práticas profissionalizantes e práticas sociocomunitárias para promover uma formação integrada, poderosa e politicamente comprometidas com a compreensão e intervenção social.

Palavras-chave

- Prática

- Integração

- Currículo

- Práxis

\section{Para citación de este artículo}

Di Franco, M. G.; Alfageme Balza, V. y Di Franco, N. (2016).

Hacia una formación integrada en la currícula universitaria: el valor de la práctica en la Universidad Nacional de La Pampa. En Revista + E versión digital, (6), pp. 180-187. Santa Fe, Argentina: Ediciones UNL. 


\section{Introducción}

Las autoras de este trabajo coordinamos actividades de extensión en el contexto de la UNLPam desde una concepción política entendida en términos de producción democrática de conocimiento y no como su mera divulgación.

En este sentido, en los últimos tres años hemos llevado adelante un proyecto que ha consolidado modalidades de intervención en el contexto escolar y comunitario con estudiantes y profesoras/es de dos facultades de nuestra universidad que forman profesoras/es. El mismo se desarrolla en el marco de la Ley 20206 de Educación Nacional y Ley 20150 Programa Nacional de Educación Sexual Integral. Se orienta hacia dos líneas: por un lado, la formación en educación sexual integral de estudiantes de escuelas secundarias a través de talleres por año centrados en las temáticas relacionadas a derechos sexuales y reproductivos, derecho a la información, violencias, prostitución, trata de personas. El abordaje integral incluye la mirada biomédica, la de género y la de los derechos ofrecidas desde la legislación internacional y nacional, tanto para formar como para interpelar discursos y prácticas hegemónicas naturalizadas. La segunda línea consiste en el acompañamiento a adolescentes embarazadas o madres en un colegio secundario, con el objetivo de sostener las trayectorias escolares, ${ }^{1}$ a través de una recuperación en proceso que finalizará con la reinserción escolar y la mejora en el aprendizaje escolar. Se organiza un plan de trabajo semanal a partir del material obligatorio que ofrecen los docentes y diseños de las/os extensionistas de la universidad pertenecientes a los Profesorados de Ciencias Biológicas, Matemática, Historia, Geografía e Inglés. El proyecto propone, de manera integrada, articular formación y extensión con el contexto de formación profesional, la cultura escolar, la detección y comprensión de las necesidades sociales y educativas y modalidades de intervención a favor de la ciudadanía.

A partir de las vivencias y aprendizajes se propone en este artículo generar reflexiones potenciadas desde la investigación acción a fin de teorizar acerca de prácticas llevadas adelante con estudiantes y docentes mientras cursan los profesorados universitarios. La hipótesis que se sostiene es que la formación profesional debe ser integrada para que favorezca la comprensión de la cultura escolar y los modos de intervención. Una formación integrada articula docencia, investigación y extensión en una episteme de praxis porque "para una teoría ciega, la practica social es invisible; para una práctica ciega, la teoría social es irrelevante" (Boaventura de Sousa Santos, 2006:16).

\section{Primeras reflexiones a partir de nuevos significados para la extensión universitaria}

En el tránsito del último siglo la extensión ha mudado significados y sentidos modificando las misiones de la universidad. ${ }^{2}$ En particular en la última década se amplifica un sentido social más profundo y democratizado, exigiendo de la universidad un compromiso claro con los campos del conocimiento académico al tiempo que con los valores sociales (Camilloni, 2015).

Esta mirada política en el acompañamiento en las trayectorias escolares de adolescentes embarazadas o mamás con su escolaridad en riesgo — por no poder cursarla escuela dado que no tienen con quien dejar sus hijas/os o deben trabajan para su sustento- nos ha puesto en acción deliberada hacia unas Prácticas de extensión.

En anteriores documentos hemos caracterizado tales prácticas (Di Franco y otros, 2013) como aquellas que:

- Se desarrollan en realidades sociales que cuestionan políticas de formación e investigación científica dominante en el campo educativo. - Se preocupan por identificar, recuperar y resguardar valiosos aportes culturales.

- ponen prioridad en prácticas educativas -en tanto prácticas vinculadas a la formación docente- se traducen en producción de conocimiento para la transformación y generan reflexiones sostenidas acerca de sus efectos formativos.

- Tienen potencialidad transformativa dado que cada decisión, posición, postura o posicionamiento hacen de su implicación política un rasgo de la práctica.

- Son prácticas relacionales -en las que participan profesores, estudiantes, profesionales de otras instituciones ciudadanas, vecinos de barrios- que desafían la construcción de intersubjetividad democrática.

- Se orientan hacia el objetivo de intentar que estudiantes, maestros, profesores, ciudadanos, "sean conscientes de que el desarrollo de los conocimientos les concierne, de que conocer es una manera de decidir lo que se tendrá en cuenta en la acción, y de que todo esto puede ayudarlos a encontrar su sitio en la historia" (Fourez, 2008:19).

- Son prácticas educativas en situaciones interculturales en tanto se objetivan "procedimientos y contenidos que desde la formación pueden tener efectos transformadores de prácticas discriminantes (o lo contrario, más impactante todavía)" (Soria, Anquín y Bellavilla, 2009:167).
1) La trayectoria escolar se define como el recorrido que sigue un alumno o un grupo de estudiantes en un tiempo determinado, desde su ingreso, estancia y egreso. Es el seguimiento que la institución hace de las actividades académicas de e cada uno/a de sus estudiantes respecto al desempeño escolar para evitar fracaso escolar. 2) Esta extensión ha variado de significado en los últimos años. García O. (UNCuyo, 2010) describe distintos momentos: en los años 60 y 70 , de plano asistencial; en los ' 80 — con el advenimiento de la democraciadifundiendo los beneficios de la distribución del capital cultural (coros, orquestas, centros culturales, los grupos de teatro) y en los ' 90 , supeditada a las reducciones presupuestarias, la Universidad consultora queda vin- culada a la tecnología. Hoy, a inicios del siglo XXI, debe rediscutirse ese significado, tanto en lo político, ético como epistemológico 


\section{6}

las prácticas de extensión llevadas

adelante han generado empatía

en las instituciones educativas

donde los estudiantes universitarios

acompañan a las mamás

\section{Nueva centralidad a las actividades de extensión}

La investigación nos ha permitido teorizar acerca de estas prácticas. En este sentido el marco legal ofrecido por el Consejo Interuniversitario Nacional (CIN) en la Resolución 692/12 plantea que:

"la comunidad académica considera que la extensión no refiere

al sólo acto de transferirlos resultados de la producción científica a la sociedad, sino 'producir' en función de las problemáticas y demandas que surgen en la vinculación de la universidad con la sociedad, por lo que debe realizarse en el marco de un proceso dialéctico en el que la sociedad y universidad se enriquezcan mutuamente nutriéndose con nuevos conocimientos y con nuevos problemas a investigar". (2012:3)

La resolución recupera en sus "Considerandos" el lugar de la universidad y afirma:

"la revalorización de la universidad como un actor más de la construcción de conocimiento y no como único protagonista de esa construcción, lo que lleva a la conformación de equipos interdisciplinarios, a la creación de espacios institucionales de la sociedad, a una nueva forma de vinculación con el contexto". (CIN, 1996:2)

No asumirse únicos protagonistas implica tener la capacidad de generar un diálogo entre conocimiento científico y otros conocimientos absolutamente fundamentales para tener un conocimiento integral y genera saberes válidos para nuestras/os estudiantes y reduce los niveles de aislamiento.

Boaventura de Sousa Santos (2010) argumenta que esto implica reconocer conocimientos que no son producidos en la universidad, sino por fuera, conocimiento valioso porque es el que organiza las prácticas sociales, la vida cotidiana de la gente. Ese conocimiento hasta ahora ha quedado fuera. La universidad se enriquece si puede atraer otros tipos de conocimientos. Este sentido político el rol de la universidad pública debe ocuparse de incorporar esos saberes populares y preocuparse por su sentido social, lo que —en la mirada del autor- puede sostenerse a través de nuevas alianzas con los movimientos sociales y en las prácticas sociales. A esto llama ecología de saberes, puesto que al abrirse va a reconocer nuevos saberes. Se favorece una alianza que protege a la universidad y lo hace porque la academia ofrece conocimientos a los movimientos y luchas sociales que permiten empoderar a la gente para sus resistencias. Esta ecología ofrece vivir y gozar con la diversidad para ampliar la comprensión de mundo.

También la UNLPam hace suyas las significaciones formuladas por el CIN y prescribe una articulación entre docencia, investigación y extensión que busca entre sus características particulares que sea "crítica, continua, concientizadora, pertinente, integradora, transformadora, creativa, formativa, participativa" (Castro, 2015:47) y constituye una de las funciones primordiales de la UNLPam. El Reglamento de Extensión aprobado en 2013 constituye el marco normativo donde se define significado y sentido de la extensión: se la define como la puesta en práctica en la comunidad, a la que pertenece, de los saberes adquiridos en la docencia y la investigación. Se proyectan los saberes construidos en cátedras universitarias, en relación con diseños curriculares, a la relación teoría-práctica, al rol de los y las profesoras, contenidos, sentido de la formación, fracaso escolar, vulnerabilidad, exclusión, etcétera. Desde la enseñanza e investigación curricular se aportan posibilidades de pensar intervenciones institucionales destinadas al aprendizaje sistemático de las capacidades para la actuación docente en las aulas y en los distintos ámbitos en donde se desempeñen profesionalmente estudiantes de los distintos profesorados. En el propio proceso de formación universitaria el vínculo con la comunidad favorece que se active una responsabilidad política gestada en la base de la universidad pública, lo cual permite vincularlos con la realidad del territorio en el que se construyen y realizar nuevos aportes e hipótesis de trabajo. El trabajo en distintas escuelas de la ciudad y de la provincia nos pone en contacto con distintas realidades, saberes disponibles respecto de la educación sexual integral, necesidades específicas, 


\title{
G6
}

\author{
sostener esta modalidad de \\ intervención modifica permanente \\ nuestra propia concepción de \\ enseñanza y aprendizaje en las \\ aulas universitarias
}

edades, experiencias, culturas vitales. En el trabajo emprendido se fortalece la noción de vinculación con el contexto en prácticas "reales", lo que nos permite consolidar este tipo de prácticas de extensión, como ya se ha anticipado. Al igual que en otras universidades, sostenemos desde la tarea en la UNLPam este argumento en tanto la extensión obliga a la intervención directa sobre un campo de acción, sobre las problemáticas reales. Esta tarea, como acción directa en el campo social, está contenida en la matriz de investigación y docencia (García y García,1997). Como práctica pedagógica —como la concebimos— puede comprenderse a partir de experiencias críticas (Woods, 1997) fundamentadas en el aprendizaje real y la vivencia comunitaria. Esta construcción es colectiva y dialógica, y en ella interactúan los conocimientos científicos con los saberes y conocimientos de la comunidad. Debería ser formulada a instancias de demandas y necesidades que permitan a la universidad cumplir con su función de anticipación teórica, su carácter innovador y compromiso social. La práctica resulta colectiva en tanto se constituye en lo que Peter Woods denomina communitas, ese esfuerzo grupal basado en la solidez del mutuo soporte y la mutua estima, y que promueve tanto el desarrollo de los coordinadores de cada experiencia local, como de los y las alumnos/as, los profesores, las familias. En las communitas los sentimientos, las habilidades, los pensamientos se comunican de manera contagiosa, donde se hace necesario la implicación de todos en una dirección compartida lo que favorece una cultura grupal. En ella los intereses compartidos como prácticas sociales pueden superar los intereses individuales (Woods, 1997:20-25). Este argumento se vuelve fundamental al trabajar la problemática para poder fortalecer la conciencia social. Es dialógica porque se sostiene sobre una comunicación lo más horizontal posible para provocar un aprendizaje abierto a partir de la activar la curiosidad epistemológica (Freire, 2000), desocultando miedos, temores, representaciones que distorsionan la comunicación y reproducen poder. Estas ideas se socializan a modo de "sentido común" y suelen ser difíciles de identificar y más aún de modificar (Giordan, 1987; Driver, 1986, 2006) si no se las pone en tela de juicio, es decir, se contraargumentan hasta tanto se las haga sucumbir. Estas ideas constituyen la cultura experiencial y resulta valioso indagarlas en puesto que es en el seno de la cultura donde se construyen significados que orientan las formas de actuar, sentir y pensar de los sujetos con relación a la naturalización de modos de violencia, fracaso escolar, invisibilidad de las problemáticas de los embarazos en riesgo. Y si bien pueden aparecer tamizadas de prejuicios, contradicciones y sin elaborar críticamente, aportan el punto de partida desde donde las personas interpretan el mundo y mueven a la acción.

Es interactiva en tanto se articulan saberes construidos como conocimiento científico —en la universidad y centros especializadosy saberes populares, propios de vivencias, sensaciones, percepciones producidas como intercambios espontáneos en la cultura vital de las personas. En esta reciprocidad se gestan mayores niveles de comunicación y comprensión.

Son prácticas que permiten desarrollar una anticipación teórica: desde la experiencia se ofrece a los estudiantes universitarios y a la comunidad educativa investigaciones relevantes de países de América Latina y el Caribe, donde la prioridad es la mirada política del curriculum, de la formación profesional, del desarrollo profesional inserto en comunidades de alta vulneración social. Tiene un carácter innovador y compromiso social. Como expresa Carbonell:

"Existe una definición bastante aceptable y aceptada que define la innovación como una serie de intervenciones, decisiones y procesos, con cierto grado de intencionalidad y sistematización, que tratan de modificar actitudes, ideas, culturas, contenidos, modelos y prácticas pedagógicas. $\mathrm{Y}$, a su vez, de introducir en una línea renovadora, nuevos proyectos y programas, materiales curriculares, estrategias de enseñanza y aprendizaje, modelos didácticos y otra forma de organizar y gestionar el currículum, el centro y la dinámica del aula". (2015:17) 


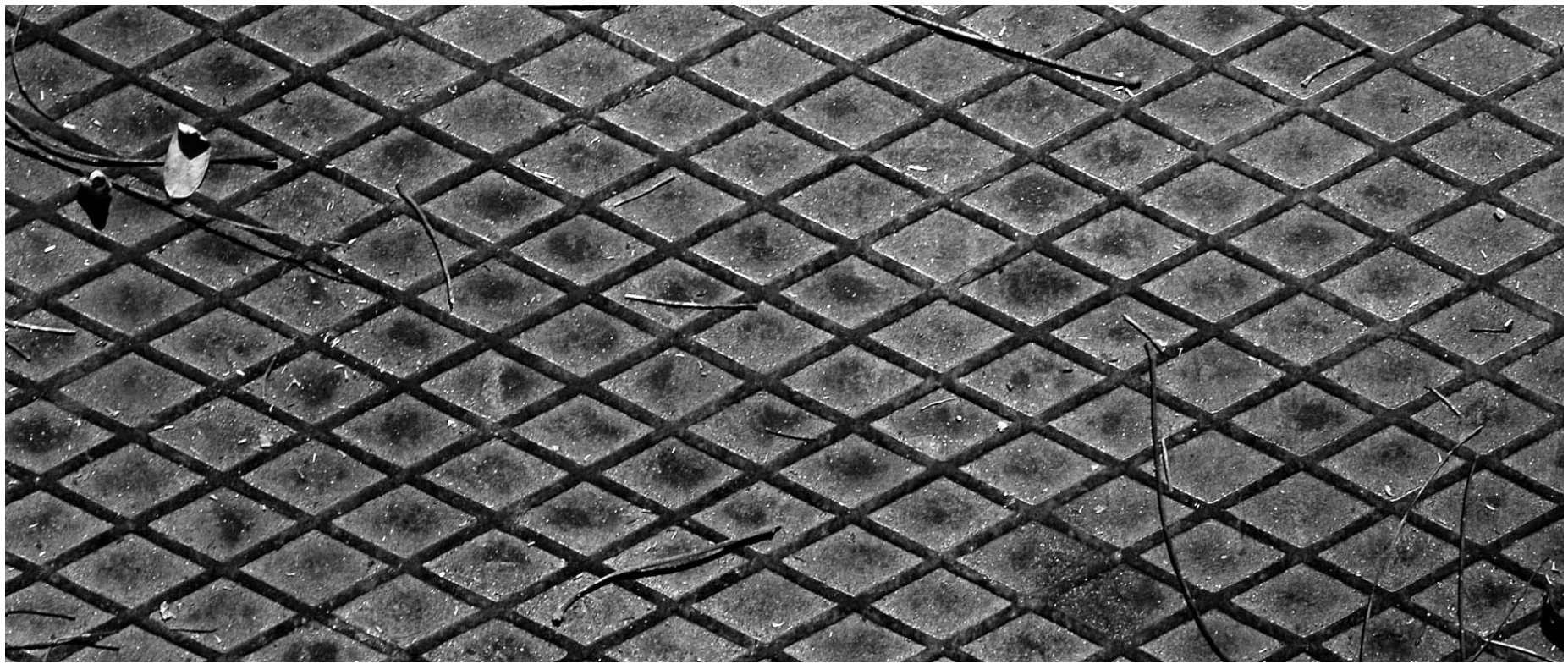

(c) Hugo Pascucci

Las prácticas de extensión llevadas adelante han generado empatía en las instituciones educativas donde los estudiantes universitarios acompañan a las mamás. El impacto sobre las biografías personales e institucionales, la valoración de retorno, el regreso desde la docencia y la investigación, la vinculación de la universidad con las escuelas, han sido vínculos innovadores en el tratamiento de estas problemáticas que requieren de urgente reconocimiento y conciencia tanto en la universidad como en la comunidad en general. Esto impactó en el compromiso asumido, se trasladó en tanto aprendizaje a los medios de comunicación local, láminas, grafitis y pasacalles, por lo que implicó una doble vía de interacción: en lo formal como proceso de aprendizaje institucional en la escuela y en su rápida socialización con el resto de la localidad.

La extensión, al ser una función sustantiva del sistema universitario, es esencial su integración —-permanente y constante— con la docencia y la investigación.

En cada acción que se genera en el trabajo por el acompañamiento y al interior de los talleres de Educación Sexual Integral buscamos preguntarnos de qué docencia hablamos al hacer extensión. Nos invitamos a pensar cómo aprende el otro, cómo generamos curiosidad, cómo recuperar saberes cotidianos, populares (lo que nos obliga a descartar aquellas posiciones abstractas, fragmentadas y alejadas de la realidad), y trabajamos en y con problemas reales a partir de una intervención interdisciplinaria integrada y relevante. Sostener esta modalidad de intervención modifica permanente nuestra propia concepción de enseñanza y aprendizaje en las aulas universitarias (Di Franco y otros, 2013).
También la investigación se modifica. El equipo interdisciplinario e interinstitucional gestado se sostiene en una investigación en la acción entendida como:

"una forma de indagación autorreflexiva que emprenden los participantes en situaciones sociales en orden a mejorar la racionalidad y la justicia de sus propias prácticas, su entendimiento de las mismas y las situaciones dentro de las cuales ellas tienen lugar" (Carr y Kemmis, 1988:174)

Estos nuevos significados de la extensión, resemantizados en la normativa y en las prácticas consolidan nuestra hipótesis provisional acerca de que debe incluirse en los curriculum a fin de aumentar los niveles de integración formativa.

\section{Curricularizar la extensión}

Desde nuestra perspectiva, toda carrera universitaria y su respectivo diseño curricular configuran una propuesta política educativa y de ella participan diferentes grupos con diferentes intereses lo cual llevará inevitablemente a que se ejerzan mecanismos de negociación (De Alba, 1998:59).

Vincular lo político con lo pedagógico ha sido parte de las reflexiones y aportes de quienes han intentado transformar la realidad con una fuerte confianza en lo educativo. En esta idea, teoría y práctica se intersectan de manera de constituirse dialógicamente. Esta postura difiere notablemente en su concepción de la lógica de la formación que deposita fuerte confianza en la teoría, dejando a la práctica suspendida hasta los últimos momentos de la formación. 
Por el contrario, en este interactuar de ambas instancias lo que pretendemos es construir e instalar un espacio formador de principios identitarios docentes a lo largo de toda la formación universitaria. Reconocer el valor formativo de la práctica habilita la inclusión de los estudiantes desde el primer año de la carrera en el contexto de su futuro desarrollo profesional, lo que favorece la comprensión de la cultura institucional y los procesos que en ella tienen lugar dándoles la posibilidad de construcción de aprendizajes relevantes.

Es en esa acción práctica, en la interacción social, en el vínculo universidad-escuela-comunidad, donde se puede advertir la naturaleza conflictiva y cargada de valor de los problemas que se presentan en el tratamiento curricular. Por ello partimos de la perspectiva que el curriculum es una herramienta en manos de las/os profesoras/es ya que pueden materializar los ideales educativos en principios de acción a partir de la propia capacidad de reflexión así como de la investigación crítica: "El curriculum es el medio a través del cual el profesor aprende porque le permite probar las ideas mediante la práctica y por tanto confiar en su juicio en vez de el de otros" (Stenhouse, 1987:160).

Esto equivale a entenderlo como hipotético, dado que obliga al profesor a explorar, a investigar para resolver los problemas que se plantean y será su tarea profesional la búsqueda de respuestas cooperativas sobre estas cuestiones en un contexto socio histórico particular para que pueda generar fisuras en la reproducción de un sistema social vigente sostenido en una narrativa coherente que le otorga sentido.

El curriculum forma a profesores y alumnos en el ejercicio deliberativo de la autonomía, el autogobierno, del desarrollo de la capacidad de comprensión a través de la utilización del conocimiento —entendiéndolo como problemático, como certeza situada-y la toma de decisiones políticamente comprometida. Es decir que al pensar al curriculum como un proyecto político es fundamental reducir los niveles de fragmentación para que práctica y teoría, dialécticamente vinculadas, constituyan la epistemología fundante de la formación universitaria. Se favorece así además la concreción de los argumentos de los planes de estudio que refieren a perfil y alcances del título y todo ello se traduce en modos de comprensión e intervención profesional en los ámbitos de formación.

\section{Prácticas de formación, de extensión y prácticas sociocomunitarias}

Ha quedado expresado en la Resolución n²40/2015 de la UNLPam el "Reglamento de Programas de Extensión Universitaria (PROEU)" que los programas de extensión: "abordan campos temáticos relacionados a las problemáticas emergentes más importantes de la agenda de interés y/o preocupación pública y consolidan los vínculos entre la Universidad y la sociedad".
Ya en la Resolución n²97/2011 por la que se crea el Programa Práctica Profesional Comunitaria en la UNLPam se habían definido a las Prácticas Comunitarias:

"Artículo $1^{\circ}$-. Se consideran Prácticas Comunitarias aquellos espacios de aprendizaje donde se ponen en juego saberes y actitudes para abordar diferentes situaciones de intervención social que propicien el contacto solidario de los estudiantes con la realidad".

Y en la Facultad de Ciencias Humanas, los planes de estudio en vigencia prescriben cuatro campos básicos de formación: disciplinar, general, pedagógica y un campo de formación denominado "Campo de la Práctica".

"Destinado al aprendizaje sistemático de las capacidades para la actuación docente en las aulas y en los distintos ámbitos en donde se desempeñe profesionalmente. Se configura como un eje transversal que apunta a resignificar los conocimientos de los otros espacios curriculares preparando a los estudiantes para una participación e incorporación progresiva en distintos contextos socioeducativos." (Resolución n 232/09)

Es decir que los y las alumnas en formación pueden participar de prácticas de formación profesional, de extensión y sociocomunitarias. $Y$ sin desconocer que las tres tienen dimensiones específicas, confluyen en el reconocimiento del valor formativo de la práctica en esa matriz dialéctica de formación. Por este motivo, para que las prácticas no sean sentidas y vividas en sentido aditivo es que se propone en este trabajo activar una pedagogía de la formación en el contexto de la responsabilidad social que enuncia la Universidad.

Estas concepciones de práctica tienen en común que indican hacia dónde se orienta el movimiento de la formación profesional: son espacios de aprendizaje en contextos situados que ponen en juego saberes ecológicos que fortalecen:

- La capacidad de intervención y acción en contextos reales complejos.

- La identificación de diversos problemas que pueden centrarse en la creciente distancia entre los ambientes de formación y los ambientes reales de las prácticas.

- La estructura de los planes de estudio - que se ha organizado en disciplinas o materias incomunicadas/inconexas, a la espera de que los alumnos integren aquello que se les enseñó en forma aisladay las prácticas de enseñanza- que tienden a que los alumnos asimilen los contenidos sin que, con frecuencia, comprendan qué valor tienen dichos contenidos respecto de sus necesidades y de la solución de los problemas de la realidad.

- Las prácticas para poder generar dispositivos de formación de mayor incidencia sociocomunitaria crítica.

- Las buenas prácticas. 


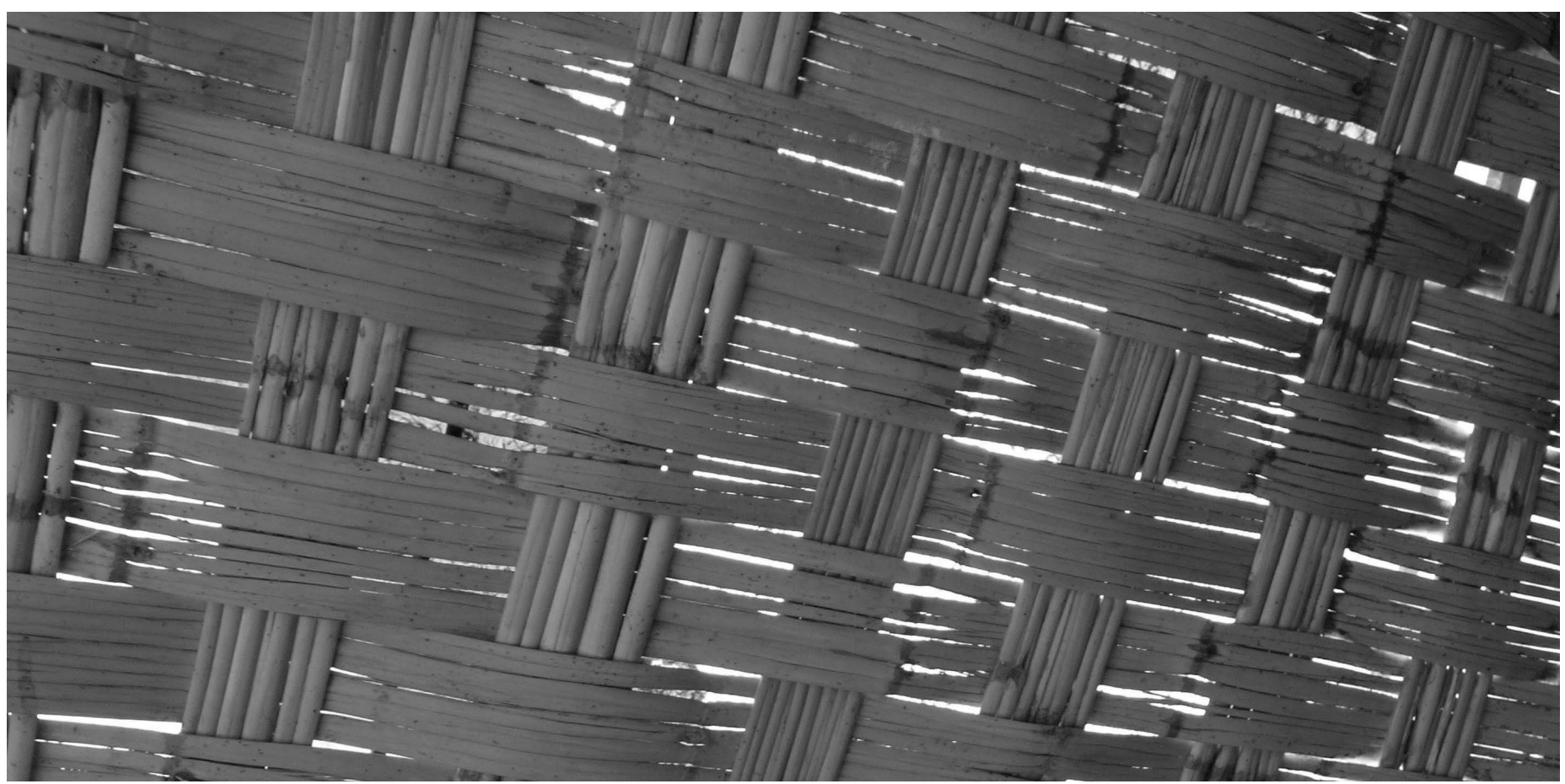

(c) Micaela Block

Unas prácticas de extensión, formación y sociocomunitarias que deberían gestarse a favor de:

- Reflexiones sistemáticas sobre la propia formación (Martínez Bonafé, 2009).

- Prácticas del Practicum, un inicio a la vida profesional en una cultura activa, investigadora, innovadora y reflexiva (Goñi, 2011:162).

- Prácticas basadas en problemas reales (Yus, 2010).

- Prácticas basadas en la cooperación y communitas (Woods, 1991; Hargreaves, 1996).

- Prácticas que involucren cuestiones cruciales con las que debe enfrentarse un profesor y la interacción/mediación entre coformadores (Sanjurjo, 2009).

- Prácticas que generen organizaciones flexibles (Hargreaves, 1996).

- Enseñanza situada (Díaz Barriga, 2006).

- Currículas integradas, globalizadas (Bernstein, 1996; Jurjo Torres, 2000, 2013).

- Prácticas que activen la investigación en la acción y la ecología de saberes (Sousa Santos, 2011).

- Prácticas que articulen cultura experiencial y académica, formación disciplinar y pedagógica, universidad y escuelas/barrio/ comunidad.

- Espacios de formación y espacios de intervención.

A modo de cierre, y buscando reconquistar la legitimidad del sentido de la universidad, podemos señalar que la formación universitaria no puede seguir pensándose de manera aislada de la sociedad.
No pueden hacerse de ese modo la producción de conocimientos, la investigación, la formación, la extensión.

Cada vez se advierte con mayor claridad que la universidad y los conocimientos que allí se producen:

"han sido un conocimiento predominantemente disciplinar, cuya autonomía impuso un proceso de producción relativamente descontextualizado con relación a las necesidades del mundo cotidiano de las sociedades". (Sousa Santos, 2011:30)

Esto ha generado varias cuestiones; el saber valorado es sólo el producido por la universidad, los saberes construidos pueden ser o no relevantes para la sociedad. Es importante poder pensar un conocimiento de límites débiles (Bernstein, 1996) difusos donde la contextualización y el trabajo en territorio potencie una negociación con otros actores, saberes, problemas. Este conocimiento pluriuniversitario, en palabras de Sousa Santos (2011:31), cuestiona fuertemente la relación ciencia, sociedad, universidad. En este proceso transita la universidad hoy, en plena discusión acerca de sentido, porque:

"La Educación Superior debe reforzar sus funciones de servicio a la sociedad, y más concretamente sus actividades encaminadas a erradicar la pobreza, la intolerancia, la violencia, el analfabetismo, el hambre, el deterioro del medio ambiente y las enfermedades, principalmente mediante un planteamiento interdisciplinario y transdisciplinario para analizar los problemas y las cuestiones planteadas". (UNESCO, 1998:3) 


\section{Referencias bibliográficas}

Bernstein, B. (1996). Pedagogía, control simbólico e identidad. Madrid: Morata. Camilloni, AR.W. (2015). Docencia, investigación y extensión: un vínculo necesario. Cuadernos de extensión universitaria. La Pampa: EDUNLPam.

Carr, W. y Kemmis, S. (1988). Teoría crítica de la enseñanza. La investigación acción en la formación del profesorado. Barcelona: Martínez Roca.

Castro, J. (2015). Orientaciones para la elaboración de proyectos y programas de extensión. Cuadernos de extensión universitaria. La Pampa: EDUNLPam.

Carbonell Sebarroja, J. (2015). Pedagogías del siglo XXI: alternativas para la innovación educativa. Madrid: Octaedro.

Consejo Universitario Nacional (1996). Resolución n 357/96.

Consejo Universitario Nacional (2012). Resolución nº 692/12.

De Alba, A. (1998). Currículum: crisis, mito y perspectivas. Buenos Aires: Miño y Dávila.

Di Franco, M.G.; Siderac, S. y Di Franco, N. (2013). Actividades científicas escolares para la escolaridad obligatoria en La Pampa. Ponencia de las XXI Jornadas de investigación de la FCH-UNLPam.

Díaz Barriga, F. (2006). Enseñanza situada, vínculo entre la escuela y la vida. México: Mc Graw Hill.

Driver, R. (1986). Psicología cognitiva y esquemas conceptuales de los alumnos. Barcelona. Enseñanza de las Ciencias, (4).

Freire, P. (2000). Pedagogía de la indignación. Cartas pedagógicas en un mundo revuelto. São Paulo: UNESP.

Fourez, G. (2008). Cómo se elabora el conocimiento. La epistemología desde un enfoque socioconstructivista. Madrid: Narcea.

García, E. y García, F. (1997). Aprender investigando. Una propuesta metodológica basada en la investigación. Sevilla: Díada.

García, O. (2010). La práctica de la Extensión como herramienta de la formación universitaria integral. Ponencia del IV Congreso Nacional de Extensión Universitaria. Universidad Nacional de Cuyo.

Giordan, A. (1987). Los conceptos adquiridos en el proceso de aprendizaje. Enseñanza de las Ciencias, (5).

Goñi, J. M. (2011). Desarrollo de una guía para la realización del practicum. En Font, V.; Planas, N.; Goñi, J.M. y Godino, J. Matemáticas. Investigación, innovación y buenas prácticas (12 Vol. III, pp. 161-174). Barcelona: Graó.
Grundy, S. (1991). Producto o praxis del currículum. Madrid: Morata.

Kemmis, S. (1988). El currículum: más allá de la teoría de la reproducción. Madrid: Morata.

Hargreaves, A. (1996). Profesorado, cultura y posmodernidad. Cambian los tiempos, cambia el profesorado. Madrid: Morata.

Martínez Bonafé, J. (1999). Trabajar en la escuela: profesorado y reformas en el umbral del siglo XXI. Buenos Aires: Miño y Dávila.

Universidad Nacional de La Pampa. (2011). Resolución del Consejo Superior n² 297/2011 sobre el Programa de "Practicas Comunitarias" (PPC). Universidad Nacional de La Pampa.

Sanjurjo, L. (2009). Los dispositivos de la formación en la práctica. Rosario: Homo Sapiens.

Soria, M.G.; Anquín, A. y Bellavilla, E. (2009). Investigación y formación docente sobre prácticas educativas situadas y contextuadas. En Yuni, J.A. La formación docente. Complejidad y ausencias. Córdoba: Encuentro Grupo Editor.

Sousa Santos, B. de (2006). La Sociología de las Ausencias y la Sociología de las Emergencias: para una ecología de saberes. En Renovar la teoría crítica y reinventar la emancipación social (encuentros en Buenos Aires). Buenos Aires: CLACSO. Sousa Santos, B. de (2010). Descolonizar el saber, reinventar el poder. Buenos Aires: Trilce.

Sousa Santos, B. de (2011). La universidad en el siglo XXI descolonizar el saber, reinventar el poder. Buenos Aires: Miño y Dávila.

Stenhouse, L. (1987). Investigación y desarrollo del currículo. Madrid: Morata. Torres Santome, J. (2000). Globalización e interdisciplinariedad: el currículum integrado. Madrid: Morata.

Torres Santome, J. (2013). El caballo de Troya, la justicia curricular. Madrid: Morata. UNESCO (1998). Declaración mundial sobre la educación superior en el siglo XXI: visión y acción. Recuperado de: http://www.unesco.org/education/educprog/ wche/declaration_spa.htm (consultado el 02/01/2017).

Woods, P. (1997). Experiencias críticas en educación. Buenos Aires: Paidós. Yus, R. (2010). Aprender haciendo. Un caso práctico en la enseñanza de las Ciencias Naturales. La nutrición de las plantas. En Pérez Gómez, A. Aprender a innovar en la práctica: procesos de innovación y prácticas de formación en la educación secundaria. Barcelona: Graó. 